\title{
RIESGOS ELÉCTRICOS
}

\section{ELECTRICAL SAFETY}

Arturo Pérez París: Universidad de Alcalá de Henares. Madrid (España) arturo.perez@mixmail.com

\section{CURRÍCULUM VITAE}

Escuela Politécnica de la Universidad de Alcalá de Henares. Ingeniero electrónico y literato. Destacan sus estudios sobre aplicaciones eléctricas y motricidad sobre los que ha publicado varios artículos científicos.

\section{RESUMEN}

La electricidad es la más poderosa fuente de energía de la que disponemos en la actualidad, ya sea de forma directa, ya sea transformada, que llega a todos los lugares. Desde finales del siglo XVIII ha existido un gremio llamado los electricistas. En sus comienzos, uno de los requisitos para entrar en este gremio era, a forma de prueba iniciática, notar el "sabor" de la electricidad a través de una descarga eléctrica. La intensidad de corriente es el factor verdaderamente peligroso en un accidente eléctrico; podríamos decir que es la que mata. Las medidas de seguridad y de prevención son muchas y muy variadas. 


\section{PALABRAS CLAVE}

Electricidad - Energía - Seguridad - Prevención

\section{ABSTRACT}

Electricity is the most powerful source of energy than currently available, either directly, be transformed, that reaches everywhere. From the late eighteenth century has been a guild called electricians. In the beginning, one of the requirements for entering this profession was, how to test initiation, notice the "flavor" of electricity through electric shock. The current is very dangerous factor in an electrical accident, we could say it's the killer. Security measures and prevention are many and varied.

\section{KEY WORDS}

Electricity - Energy - Safety - Prevention

\section{ÍNDICE}

1. Introducción

2. Factores de riesgo

3. Medidas de seguridad

4. Primeros auxilios

5. Dedicatoria 


\section{TEXTO:}

\section{Introducción}

Cuenta la historia que, hacia finales del siglo XVIII, existía un gremio llamado de los electricistas. Hablamos de los tiempos de Franklin. Estos electricistas tenían poco que ver con los actuales profesionales, así como el concepto de átomo que tenían los griegos poco se asemeja al nuestro. Uno de los requisitos para entrar en este gremio era, a forma de prueba iniciática, notar el "sabor" de la electricidad a través de una descarga eléctrica. Afortunadamente, estas prácticas están hoy en día en desuso. De otra forma, más de uno o una en mi profesión hubiera salido mal parado, o quizás, quién sabe, se le habrían curado todos los males o, cuando menos, algunos.

Bromas aparte, la electricidad es la más poderosa fuente de energía de la que disponemos en la actualidad; ya sea de forma directa, ya sea transformada, llega a todos los lugares y difícilmente podemos encontrar algún caso en el que no se tenga una relación o dependencia directa con ella.

El motivo de la redacción del presente artículo responde a la necesidad de exponer estos riesgos, no para que temamos a esta maravillosa fuente energética, sino más bien para que la respetemos. Debemos tener en cuenta que el agua es un excelente conductor de la electricidad y, dado que el ser humano tiene en su composición un porcentaje aproximado al $80 \%$ de su peso en forma de agua, resulta obvio pensar que esté expuesto a un sin fin de riesgos derivados de la manipulación y contacto con la electricidad.

Para obtener una idea de su peligrosidad, podemos indicar que por cada 200.000 habitantes se produce un accidente mortal, debido a la electricidad. Aun así, es una cantidad cincuenta veces inferior al índice de accidentes de tráfico en un núcleo 
también de 200.000 habitantes. En el mundo laboral, los accidentes eléctricos suponen el $0,35 \%$ de los accidentes que causan baja laboral, el 0,90\% de los accidentes que implican incapacidad permanente y el $4 \%$ de los accidentes mortales.

\section{Factores de riesgo}

Son muchos los factores que influyen en el ser humano que sufre un accidente laboral por una descarga eléctrica. Entre ellos, los más comunes son los siguientes:

- Intensidad de la corriente.

- Tensión o voltaje de la corriente.

- Resistencia del cuerpo humano al paso de la corriente.

- Frecuencia de la corriente.

- Tiempo de exposición o de contacto.

- Trayectoria del paso de la corriente por el organismo.

- Tipo de corriente.

- Sexo del accidentado.

Es la conocida ley de Ohm, la que nos va a explicar la importancia de estos factores:

Para una Resistencia que se suponga constante, la Intensidad de la corriente que pasa entre dos puntos de un conductor es directamente proporcional a la diferencia de potencial (tensión) entre esos dos mismos puntos.

$$
R=\frac{V a-V b}{I}
$$

Donde:

$\mathrm{I}=$ Intensidad de la corriente, medida en amperios. 
$\mathrm{Va}-\mathrm{Vb}=$ Tensión o diferencia de potencial medida en Voltios.

$\mathrm{R}=$ Resistencia, medida en Ohmios.

La intensidad de corriente es el factor verdaderamente peligroso en un accidente eléctrico; podríamos decir que es la que mata. Junto con el tiempo de contacto, es quizá el factor más importante de los reseñados con anterioridad. A partir de 0,01 Amperios, es peligrosa y resulta mortal a partir de 0,10 Amperios. En cantidades intermedias puede producir desde contracciones musculares, hasta fibrilación ventricular, pasando por estadios intermedios de parálisis temporales, tanto cardíacas como respiratorias

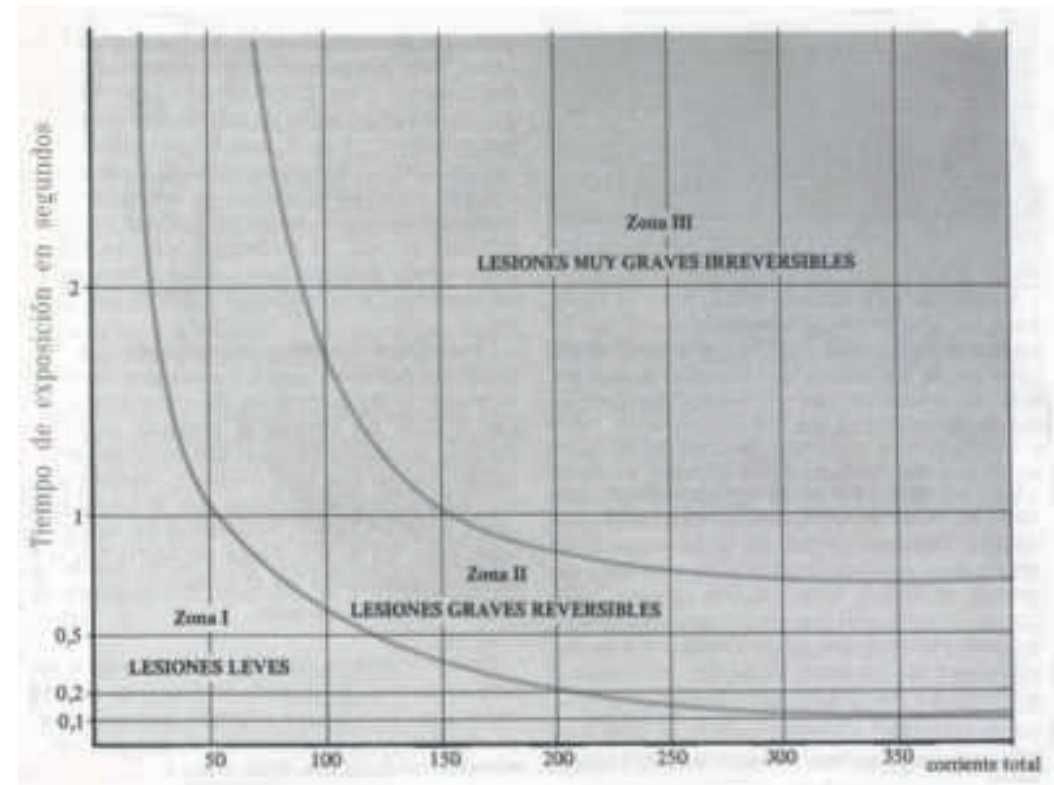

Podemos determinar como umbral de percepción de la corriente, 0,003 Amperios, ya que, con cantidades inferiores a ese valor, el contacto eléctrico puede ser perfectamente soportado sin peligro alguno.

La tensión o voltaje de la corriente depende fundamentalmente de las características de la instalación. En general, podemos decir que existirá un riesgo mayor cuanto mayor sea la tensión, atendiendo a la Ley de $\mathrm{Ohm}$, dada que la resistencia de nuestro 
cuerpo se mantendrá más o menos constante y, por tanto, a mayor tensión, mayor corriente.

La resistencia es un factor de gran variabilidad. Ésta puede variar en el ser humano entre los 500 y 100.000 Ohmios, considerando como cantidades normales los comprendidos entre 1.000 y 2.000 Ohmios. Estas diferencias están en función de las características fisiológicas del cuerpo humano, por lo que varía de unas personas a otras, no pudiéndose dar unas cantidades idénticas para todos los casos.

La resistencia de contacto de la piel es muy variable así lo indican estudios estadísticos que han demostrado que, de cinco accidentes eléctricos, tres tienen lugar a lo largo de los cinco meses más calurosos del año. También se ha determinado que una piel seca puede presentar una Resistencia de algunos cientos de miles de Ohmios, mientras que en casos de máxima sudoración, ésta baja hasta los 1.000 Ohmios escasos.

La resistencia de los tejidos internos del cuerpo humano también varía, ya que está en función del recorrido que la corriente realice por el mismo; no obstante, este valor puede calcularse entre valores comprendidos de 100 a 5.000 Ohmios.

Si relacionamos la tensión de la corriente con la Resistencia del cuerpo humano, vemos que ésta fluctúa en función de aquélla; como ejemplos podemos dar las siguientes cantidades: 


\begin{tabular}{|c|c|}
\hline $\begin{array}{c}\text { Tensión } \\
\text { (voltios) }\end{array}$ & $\begin{array}{c}\text { Resistencia } \\
\text { (Ohmios) }\end{array}$ \\
\hline 24 & 10.000 \\
\hline 65 & 3.000 \\
\hline 150 & 2.000 \\
\hline
\end{tabular}

A partir de esos últimos 150 Voltios, la resistencia es prácticamente constante hasta que se produce la herida en la piel, ya que, a partir de ese momento, la resistencia que ofrece el cuerpo humano es la que ofrecen los tejidos internos, como hemos comentado anteriormente.

Vamos a considerar un ejemplo sencillo de aplicación de la ley de Ohm, en el que, manteniendo una tensión constante, y variando la resistencia del cuerpo, se producen intensidades diversas. De esta manera, la gravedad del accidente, resultante del contacto eléctrico, estará en función de la resistencia óhmica que opongamos; así podemos deducir las condiciones de seguridad que debería reunir cualquier persona al realizar su trabajo sin riesgos de electrocución. Vamos a suponer una Tensión de 220 Voltios, que es la más comúnmente utilizada:

\begin{tabular}{|c|c|c|c|}
\hline $\begin{array}{c}\text { Tensión } \\
\text { (Voltios) }\end{array}$ & $\begin{array}{c}\text { Resistencia } \\
\text { (Ohmios) }\end{array}$ & $\begin{array}{c}\text { Intensidad } \\
\text { (Amperios) }\end{array}$ & Calificación \\
\hline 220 & 1000 & 0,22000 & Mortal \\
\hline \multirow{2}{*}{220} & 10000 & 0,02200 & Grave \\
\hline \multirow{2}{*}{220} & 20000 & 0,01100 & \\
& 30000 & 0,00733 & Tolerable \\
& 40000 & 0,00550 & \\
& 50000 & 0,00440 & \\
\hline
\end{tabular}




\begin{tabular}{|l|l|l|l|}
\hline \multirow{2}{*}{220} & 75000 & 0,00293 & Sin peligro \\
& 80000 & 0,00275 & \\
90000 & 0,00244 & \\
& 100000 & 0,00220 & \\
\hline
\end{tabular}

Del estudio de este cuadro concluimos que, para una tensión de 220 Voltios, deberemos ofrecer una resistencia mínima de seguridad superior a los 75.000 Ohmios, que sólo será alcanzada por personas de mínima sudorización y de piel resistente (es decir, que tengan callosidades) con un sistema nervioso equilibrado y que no sufra ninguna enfermedad crónica en las vísceras fundamentales. Si esto no es así, deberá protegerse bien con prendas de protección personal homologadas (como guantes, botas, etc.) o bien aislarse del suelo mediante banquetas o alfombrillas aislantes.

Observamos también, corroborando lo anteriormente expuesto, que con el aumento de la resistencia va disminuyendo el peligro $y$, dado que cuando se manipule un circuito eléctrico normalmente no podrá actuarse sobre el voltaje, ya que éste será constante, nuestra actuación deberá estar encaminada, casi de forma exclusiva, a aumentar la resistencia del cuerpo humano para que, de esta forma, disminuya la intensidad, reduciendo así el peligro de electrocución.

Otro factor a tener en cuenta es la frecuencia de la corriente. En España es frecuente una frecuencia de 50 ciclos por segundo (Hercios), aunque aumenta en algunas ocasiones, como por ejemplo, en aparatos médicos que llega a los 1.000 ciclos por segundo. A medida que la frecuencia aumenta, la corriente se hace menos peligrosa ya que se produce el llamado efecto pelicular (la corriente circula, preferentemente, por la superficie de la piel sin penetrar en órganos internos). 
El tiempo de exposición es otro de los factores fundamentales en la peligrosidad de las descargas eléctricas, dado que la intensidad, antes comentada, tiene un carácter acumulativo, y de ahí la relación con el tiempo durante el cual esa intensidad actúa sobre el cuerpo humano. Éste admitirá menor Intensidad, cuanto mayor sea el tiempo de exposición. La Organización Internacional del Trabajo (O. I. T.), con sede en Ginebra, marca la Intensidad que puede admitir el ser humano, medida en miliamperios (mA), mediante la fórmula:

$$
I=\frac{60}{\sqrt{t}}
$$

siendo "t" un valor siempre inferior a los 3 segundos.

El riesgo mayor al que se somete al ser humano, al contacto con la electricidad, es el de la fibrilación cardiaca, que suele ser mortal, si no se actúa con rapidez sobre el paciente, efectuándole una correcta recuperación cardiorrespiratoria. La fibrilación ventricular consiste en una serie de contracciones totalmente arbitrarias que realiza el músculo cardiaco. Existe una relación entre el tiempo de exposición a la corriente y la Intensidad, lo que da como resultado la fibrilación. Se podrá producir fibrilación ventricular, si el tiempo de exposición a una corriente eléctrica se mantiene a lo largo de un periodo cuya duración mínima sea inversamente proporcional a la intensidad de la corriente. Las cifras que se barajan en este sentido varían, según la investigación consultada sobre este tema, pudiendo aceptarse como válidas las que a continuación exponemos:

\begin{tabular}{|c|c|}
\hline $\begin{array}{c}\text { Corriente } \\
\text { (miliamperios) }\end{array}$ & $\begin{array}{c}\text { Tiempo } \\
\text { (segundos) }\end{array}$ \\
\hline 15 & 120 \\
\hline 20 & 60 \\
\hline 30 & 35 \\
\hline 50 & 2 \\
\hline
\end{tabular}




\begin{tabular}{|c|c|}
\hline 100 & 1,5 \\
\hline 500 & 0,11 \\
\hline 1000 & 0,03 \\
\hline
\end{tabular}

Se considera que no existe fibrilación a nivel general, cuando el tiempo de exposición es inferior a 0,2 segundos. Este tiempo se considera, a su vez, inferior al correspondiente entre dos sístoles consecutivas, es decir, en diástole, pero debe tenerse en cuenta que, si es coincidente con la llamada «fase crítica», sí puede existir fibrilación, tal y como se muestra en la Fig. 2. Esto se ha podido determinar a través de diversas experiencias realizadas por entidades competentes.

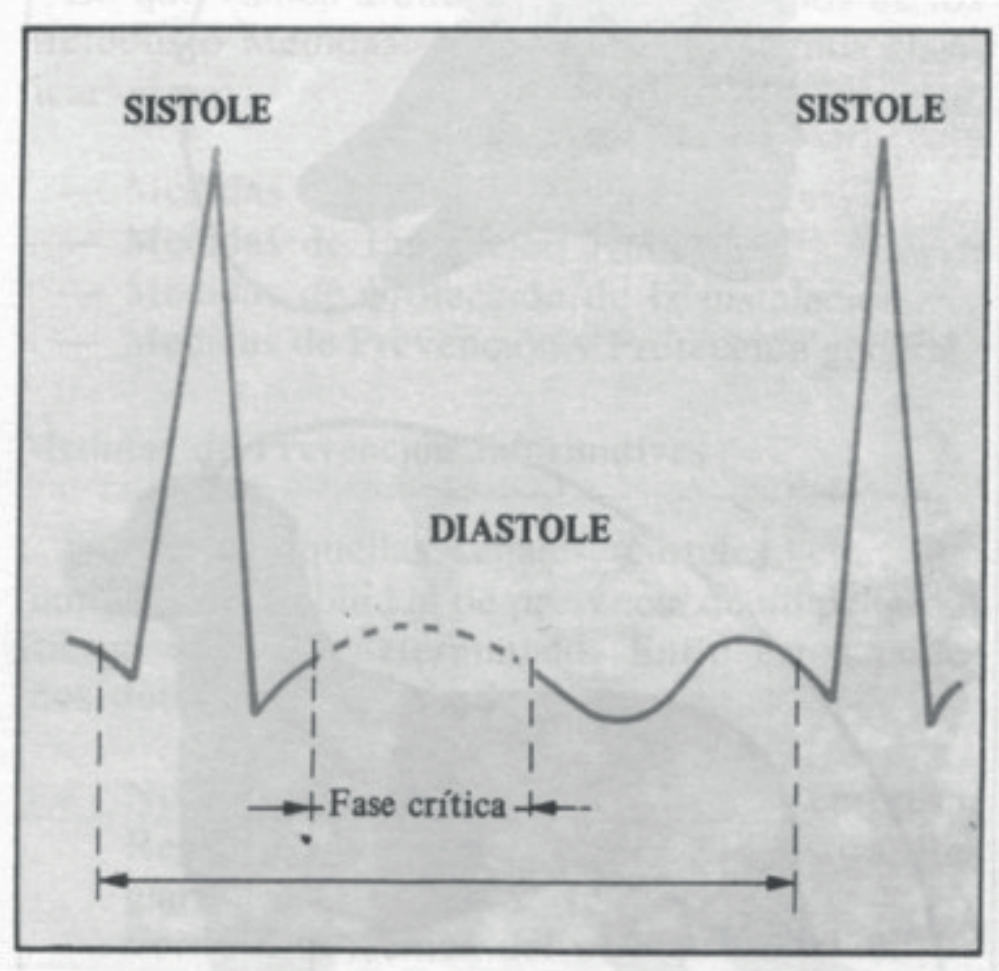

Además de lo ya expuesto, un factor primordial a tener en cuenta es la trayectoria del paso de la corriente por el organismo. Ésta es un factor influyente en la gravedad de la lesión. Se considera que la gravedad es máxima cuando el recorrido pasa a través 
de vísceras fundamentales del cuerpo humano, como pueden ser el corazón, el cerebro, etc. La confirmación de este hecho viene determinada por el conocido experimento de Weis. Consiste en la siguiente prueba: A un perro de consistencia media se le hizo pasar una corriente de $400 \mathrm{~mA}$ entre el maxilar inferior y el cráneo, sufriendo una parada respiratoria de la que se recuperó con facilidad. Más tarde se le hizo pasar la misma Intensidad de corriente, pero variando los puntos de contacto, haciéndola circular entre el cráneo y una de sus patas; en esta ocasión, el animal murió de forma instantánea por fibrilación. Aunque este experimento sea una completa salvajada, podemos concluir que los recorridos más peligrosos de la corriente eléctrica en el cuerpo humano son los que ponen en contacto una de las manos con la cabeza, o bien con el pie contrario; o bien la corriente entra por una de las manos, atraviesa el tórax y sale por la otra mano. 


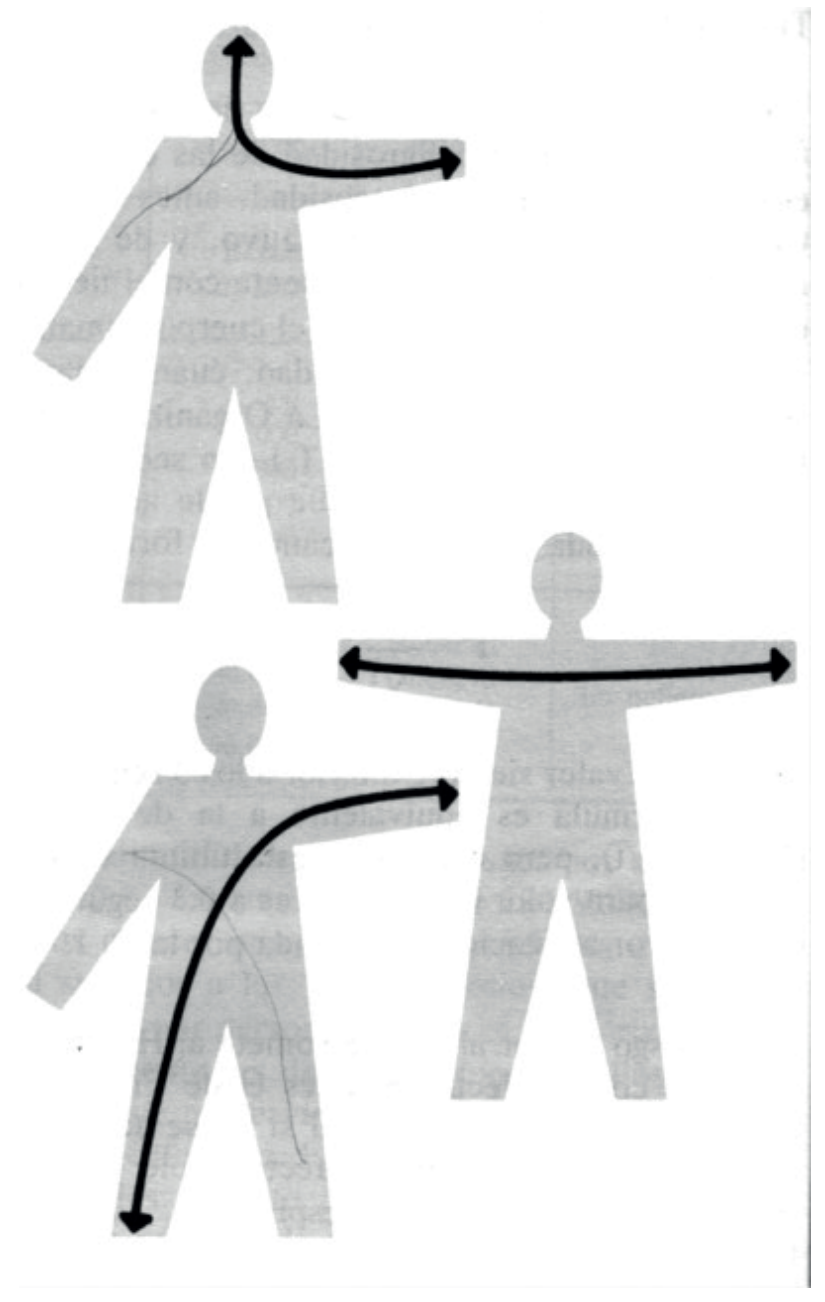

La corriente eléctrica puede ser de dos clases: Alterna y Continua. En la primera cambiará de sentido (tantas veces por segundo) en función de la frecuencia que tenga. La segunda circula siempre en la misma dirección. La peligrosidad de la corriente está en función de la Frecuencia, ya que una corriente alterna de baja frecuencia (inferior a $1.000 \mathrm{~Hz}$ ) produce efectos superiores a la misma intensidad de corriente continua, mientras que para frecuencias superiores a los $20.000 \mathrm{~Hz}$ se invierte su peligrosidad.

Los tipos de contactos eléctricos que puede haber son los siguientes:

-Directo. 
-Indirecto.

Se entiende por contacto eléctrico directo, cuando el cuerpo humano es atravesado por la corriente eléctrica, al interrumpir éste el paso de aquella por elementos que están en tensión, tales como cables sin protección, interruptores en mal estado, etc. Puede decirse que en este caso, el cuerpo humano entra a formar parte del circuito eléctrico.

Se entiende por contacto eléctrico indirecto, la desviación de la corriente eléctrica a través de otro cuerpo, al qué se denomina masa, y de forma casual y fortuita el cuerpo humano entra en contacto con dicha masa. De esta forma, se realiza también un nuevo circuito eléctrico.

El contacto eléctrico indirecto es el más común e imprevisto, ya que el directo es más difícil de que ocurra, puesto que la deficiencia está a la vista del operario, lo que le hará aumentar su precaución. No obstante, ambos casos pueden ser evitados con el uso de las medidas de prevención adecuadas. No debe olvidarse que en todos los casos el cuerpo humano es puente por el que fácilmente puede pasar la corriente eléctrica en su camino a tierra y por ello deberá ser fuertemente protegido o bien establecer otro puente que favorezca el camino de forma más fácil. Esto se consigue con los medios de prevención y protección.

\section{Medidas de seguridad}

Aunque las medidas de seguridad para casos de electrocución podrían darse de forma individualizada para los contactos directos y los contactos indirectos, en la gran mayoría de los casos éstas servirán para ambos tipos de contactos. A continuación, exponemos una clasificación de las medidas o tipos de los métodos:

- Medidas de Prevención Informativas. 
- Medidas de Protección Humana.

- Medidas de Protección de la instalación.

- Medidas de Prevención y Protección general.

Las Medidas de Prevención informativas, son todas aquellas señales, carteles, etc., que indiquen la posibilidad de presencia de un peligro o Riesgo eléctrico determinado. Entre éstos podemos determinar los siguientes:

- Normas de Seguridad: De la propia empresa. Reglamentos del Ministerio de Industria.

- Reglamentos de la O. I. T.

- Formación técnica del personal afectado.

- Señalización: de Prohibición, de Precaución, de Obligatoriedad, de Información, etc.

Las Medidas de Protección Humana, se refieren al conjunto de herramientas y prendas o ropa de trabajo, que se utilizan de forma individualizada, por parte de cada operario, con el fin de aumentar su Resistencia y, por consiguiente, disminuir el riesgo. Entre ellas destacamos:

o Plataformas y taburetes aislantes.

○ Alfombrillas.

- Guantes de caucho y goma aislantes.

- Calzado aislante.

- Casco.

- Pértigas de maniobra.

- Pantallas y gafas.

o Herramientas con aislamiento. 
Las prendas de protección personal no deben ser consideradas suficientes por sí solas, aunque sí necesarias, debiéndose siempre tomar otras medidas de seguridad que las complementen.

Las Medidas de Protección de la Instalación son básicamente:

-La Puesta a tierra de las masas. Esta protección consiste en la unión, por medio de un conductor, de las partes metálicas de una instalación eléctrica, que no estén bajo tensión, con el terreno; de forma que al ofrecer una Resistencia muy baja, facilite el paso de la corriente respetando el cuerpo humano. En lugares donde existe cantidad de motores o elementos eléctricos, podrán ponerse muchas puestas a tierra, pero deberá procurarse de que estén muy separadas o bien que estén al mismo potencial, si están muy próximas unas de otras (conexiones equipotenciales).

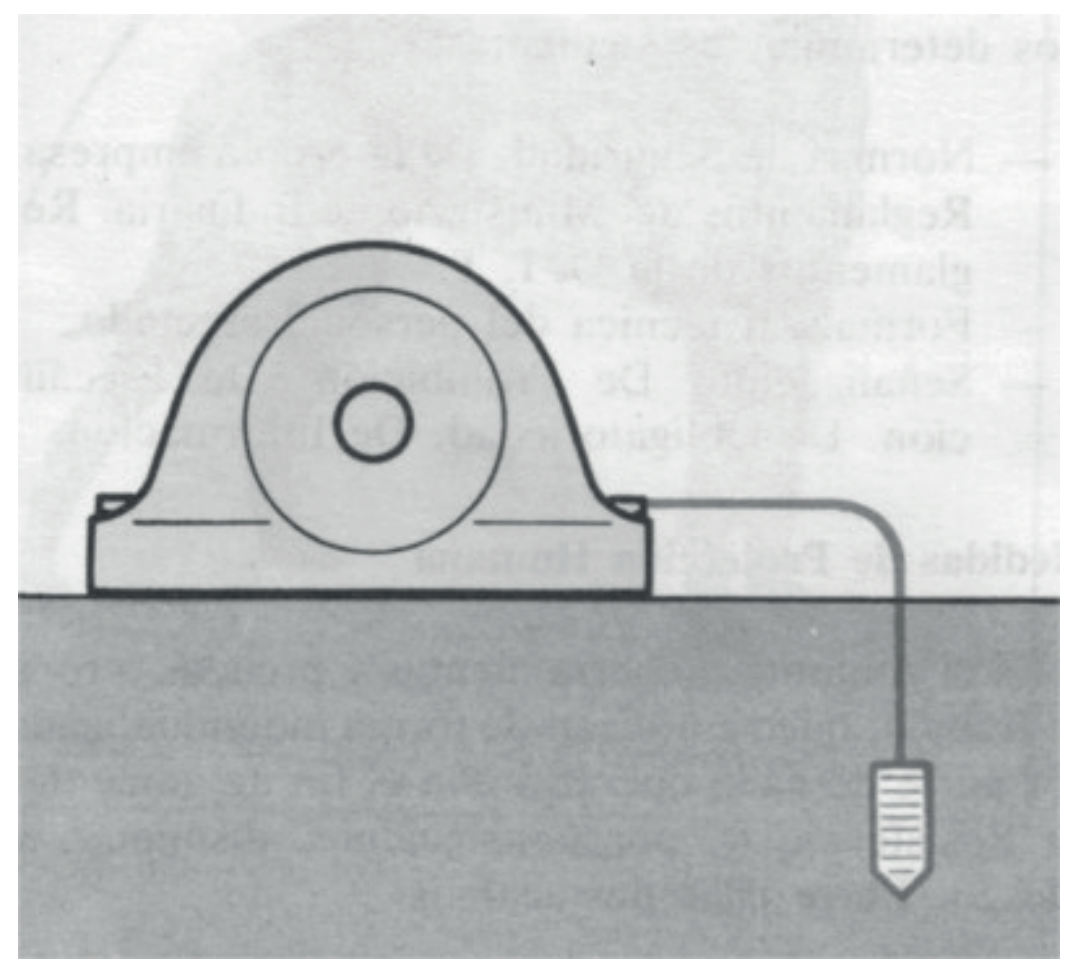

-Recubrimiento de masas accesibles. Es un procedimiento costoso pero de gran utilidad, ya que obliga de forma periódica a realizar inspecciones en la instalación. 
-Separación entre partes activas y masas accesibles. Este procedimiento también se conoce como aislamiento de protección o doble aislamiento. Cuando se utilice, no deberá colocarse puesta a tierra y, las máquinas, sometidas a esta protección, deberán estar señalizadas. Este sistema consiste en la colocación de un aislamiento suplementario al funcional, con el fin de proteger la masa de una posible corriente de defecto producida por la primera.

-Disminución de tensiones. Este procedimiento consistente en disminuir la tensión para que la Intensidad que circule a través de quien entre en contacto sea lo menos peligrosa posible. Éstas podrán ser de 15 y 24 Voltios para corriente alterna. Tiene muchas limitaciones en su uso, debido a su coste y la potencia que hoy día exigen las instalaciones. Suele emplearse en quirófanos, circuitos de mandos, juguetes, lámparas portátiles, etc.

-Fusibles e interruptores magnetotérmicos. Estos no deberían considerarse como Dispositivos de Seguridad propiamente dichos, si bien protegen a las máquinas de forma conveniente. Así pues, pueden considerarse como protecciones de acompañamiento. El fusible deberá calcularse al doble de la intensidad nominal, para evitar que se rompa de forma continuada.

-Interruptores diferenciales. Estos son dispositivos automáticos de corte del paso de la corriente cuando se presenta una fuga. Un factor importante es su sensibilidad, que debe ser adecuada para cada caso. A mayor sensibilidad, la toma de tierra será más basta, ya que las máquinas tienen toma de tierra natural.

Otras medidas de prevención y protección General serán:

- Protección con carcasas de todos los interruptores, seccionadores, etc.

- Aviso de reparación de línea, para evitar meter tensión de forma accidental.

- Enterramiento de las líneas de conducción. 
- Extremar medidas de Seguridad en locales con una humedad relativa superior al $70 \%$ y en locales en los que se manipulen materiales muy inflamables, tales como explosivos, petróleos, etc.

Otro riesgo eléctrico muy común es el derivado de la electricidad estática. Entendemos por ésta, aquella que se produce cuando se frotan dos sustancias de diferente constante dieléctrica y una de las cuales, cuando menos, no es buena conductora. Resulta fácil de comprender que este fenómeno se produce con cierta facilidad y en numerosas ocasiones, por lo que los riesgos que se derivan de ella son, asimismo, muy elevados. Como ejemplos de producción de electricidad estática podemos señalar el transporte de fluidos por tuberías o cisternas, en la fabricación de papel en rollos, en máquinas en las que existan correas o cintas de cuero, en el pintado de objetos metálicos por medio de pistola de pulverización, etc.

La electricidad estática es fuente de innumerables problemas que pueden presentarse incluso en nuestros hogares con consecuencias desagradables. Pensemos en la siguiente situación: cuando al cerrar la puerta de un coche después de un viaje, sufrimos una descarga eléctrica, nos hemos convertido en un puente entre la carga de electricidad del coche, acumulada por su contacto con el aire, a alta velocidad, y la tierra. Las medidas preventivas que deben de tenerse en cuenta son las siguientes:

$\checkmark$ Adecuada puesta a tierra para máquinas, pero sobre todo, para cisternas de almacenamiento y transporte de fluidos.

$\checkmark$ Adhesivos especiales para correas de transmisión.

$\checkmark$ Establecer una buena ventilación para que los vapores inflamables puedan ser evacuados con facilidad.

$\checkmark$ Efectuar un mantenimiento adecuado en los locales en los que existe peligro de humedades relativas altas.

$\checkmark$ Ionización del aire por medio de sustancias radiactivas, alta tensión o inducción. No obstante, aunque son muy eficaces estos procedimientos, 
no podemos ocultar el alto coste que supone su implantación, así como sus problemas técnicos.

\section{Primeros auxilios}

Después de haber descrito los conceptos básicos sobre las posibles fuentes de riesgo eléctrico, procederemos a exponer los primeros auxilios que deberemos aplicar, siempre midiendo nuestra forma de actuar, para evitar agravar las lesiones o causar unas nuevas al accidentado, a otras personas o a nosotros mismos. Precisamente en el auxilio a una víctima de accidente eléctrico es donde hemos de extremarse todas las medidas, para evitar que nuestra actuación sea desafortunada.

Nunca debemos olvidar que el cuerpo humano es un buen conductor de la electricidad, como lo son el agua y los objetos metálicos. Antes de intentar cualquier ayuda a una víctima de la electricidad es totalmente necesario tener la seguridad de que éste no está en contacto con un conductor en tensión. En caso contrario, tocar a la víctima sin precauciones nos ocasionaría que sufriéramos las mismas lesiones, al igual que, si por estar ardiendo sus ropas, tratamos de arrojarle agua o separarlo con instrumento no suficientemente aislador de la corriente.

Para desprender a la víctima, en caso de haber quedado suspendida de un cable, debemos pensar que nosotros solos no vamos a poder manejarla, por lo que hemos de tomar medidas especiales para que, en su caída no se produzcan fracturas.

En los accidentes por electricidad hay que distinguir entre dos tipos de corriente: la natural atmosférica y la producida artificialmente. El tratamiento es prácticamente igual en una que en otra y lo único que varía son las medidas de prevención de accidentes. Nosotros, desde el punto de vista del socorrista, nos referimos únicamente a la artificial, que es la que más veces requerirá nuestra ayuda. 
Nuestra primera actuación siempre ha de ser cortar inmediatamente la corriente, si el aparato de corte o los fusibles son fácilmente accesibles. En caso de no poder llegar a estos aparatos por encontrarse distantes, se procurará poner los conductores en cortocircuito, procurando para ello quedarse fuera del alcance de la corriente y lanzando una barra de hierro o cadena que ponga en contacto ambos conductores para hacer saltar los fusibles y conseguir el mismo efecto.

En la electricidad industrial habrá que distinguir entre la de alta y baja tensión. La de alta tensión, causa menos lesionados, porque existe gran conciencia del peligro. Por lo general la manipulan personas muy adiestradas, pero, no obstante, puede existir el caso de un cable caído en el suelo y que transmita la corriente, debiendo tener en cuenta que, en estos casos, no sólo su contacto, sino la simple aproximación a corta distancia puede hacer saltar el arco y por simple deslumbramiento causarnos lesiones oculares. Es frecuente que estas corrientes, como consecuencia de la descarga, lancen la víctima a distancia, provocándole fracturas que, en ocasiones, son las únicas consecuencias y, en otras, una complicación más de las lesiones que se producen. Las descargas de alta tensión suelen producir parálisis cardiorrespiratoria, y suelen tardar mucho tiempo en recuperarse, pudiendo dar también manifestaciones tardías, por lo que las víctimas deben estar varios días en observaciones bajo control médico.

La de baja tensión también es muy peligrosa y es causa de muchas muertes, sin olvidar que a ésta estamos expuestos prácticamente todos, ya que está presente en nuestros hogares, y con la que además, por la falsa creencia de que no es peligrosa, se cometen múltiples imprudencias, desde utilizarla pulsando interruptores o manipulando enchufes estando con todo nuestro cuerpo o parte de él en contacto con el agua o la humedad (hemos dicho es muy buena conductora), hasta no mandar reparar y revisar los electrodomésticos cuando vemos que al utilizarlos dan 
pequeños hormiguillos, evidencia de algún contacto o alguna reparación defectuosamente hecha.

Las víctimas de estas corrientes suelen quedar agarrotadas y pegadas al conductor, ya que se produce una estimulación nerviosa que ocasiona contracción espástica de los músculos flexores de las manos y brazos. Ésta, a veces, persiste después de cortada la corriente, por lo que debemos desagarrotar las manos.

En ocasiones la parada cardiaca está motivada por la presencia de una fibrilación ventricular (contracciones parciales de los ventrículos, rapidísimas y descoordinadas, totalmente ineficaces para mantener la circulación de la sangre), cuando el paso de la corriente ha cogido de lleno el eje cardíaco.

Otras veces por descarga en el bulbo puede producirse la parálisis respiratoria. También en esta baja tensión en ocasiones puede salir despedida la víctima, ocasionándosele fracturas.

Esperamos sinceramente que esta nota haya servido para poner en aviso a todos aquellos que manejan la electricidad con gran alegría, sin pensar en las consecuencias de un uso inadecuado de las medidas de protección.

\section{Dedicatoria}

Quisiera dedicar este trabajo a Ana Belén, mi amor, mi compañera, mi amiga y un montón de cosas más que con palabras no puedo expresar. Cuando todo estaba oscuro y no sabía dónde ir -quizás estaba ciego y no veía el camino-, me tendió su mano para afrontar juntos un futuro lleno de esperanzas e ilusiones, de las cuales yo ni siquiera tenía noticias. 
